Check for updates

Cite this: Phys. Chem. Chem. Phys., 2021, 23, 9457

Received 29th January 2021, Accepted 31st March 2021

DOI: 10.1039/d1cp00429h

rsc.li/pccp

\section{Pulse sequence and sample formulation optimization for dipolar order mediated ${ }^{1} \mathrm{H} \rightarrow{ }^{13} \mathrm{C}$ cross-polarization $\dagger$}

\author{
Stuart J. Elliott, (D)*a Olivier Cala, ${ }^{a}$ Quentin Stern, ${ }^{a}$ Samuel F. Cousin, ${ }^{a b}$ \\ Dmitry Eshchenko, ${ }^{c}$ Roberto Melzi, ${ }^{d}$ James G. Kempf ${ }^{e}$ and Sami Jannin ${ }^{a}$
}

\begin{abstract}
We have recently demonstrated the use of contactless radiofrequency pulse sequences under dissolution-dynamic nuclear polarization conditions as an attractive way of transferring polarization from sensitive ${ }^{1} \mathrm{H}$ spins to insensitive ${ }^{13} \mathrm{C}$ spins with low peak radiofrequency pulse powers and energies via a reservoir of dipolar order. However, many factors remain to be investigated and optimized to enable the full potential of this polarization transfer process. We demonstrate herein the optimization of several key factors by: (i) implementing more efficient shaped radiofrequency pulses; (ii) adapting ${ }^{13} \mathrm{C}$ spin labelling; and (iii) avoiding methyl group relaxation sinks. Experimental demonstrations are presented for the case of $\left[1-{ }^{13} \mathrm{C}\right]$ sodium acetate and other relevant molecular candidates. By employing the range of approaches set out above, polarization transfer using the dipolar order mediated cross-polarization radiofrequency pulse sequence is improved by factors approaching $\sim 1.65$ compared with previous results. Dipolar order mediated ${ }^{1} \mathrm{H} \rightarrow{ }^{13} \mathrm{C}$ polarization transfer efficiencies reaching $\sim 76 \%$ were achieved using significantly reduced peak radiofrequency pulse powers relative to the performance of highly sophisticated state-of-the-art cross-polarization methods, indicating ${ }^{13} \mathrm{C}$ nuclear spin polarization levels on the order of $\sim 32.1 \%$ after 10 minutes of ${ }^{1} \mathrm{H}$ DNP. The approach does not require extensive pulse sequence optimization procedures and can easily accommodate high concentrations of ${ }^{13} \mathrm{C}$-labelled molecules.
\end{abstract}

\section{Introduction}

Conventional nuclear magnetic resonance (NMR) techniques are limited by an intrinsic lack of sensitivity and generate weak signals. Dissolution-dynamic nuclear polarization (dDNP) has proven to be a widely applicable hyperpolarization method to intensify detectable NMR signals from a number of small molecules and materials by up to four orders of magnitude, ${ }^{1}$ with far-reaching applications in clinical research. ${ }^{2-4}$ Adequate $\mathrm{dDNP}$ of dilute insensitive nuclear spins such as ${ }^{13} \mathrm{C}$ at low

\footnotetext{
${ }^{a}$ Centre de Résonance Magnétique Nucléaire à Très Hauts Champs - FRE 2034 Université de Lyon/CNRS/Université Claude Bernard Lyon 1/ENS de Lyon, 5 Rue de la Doua, 69100 Villeurbanne, France.

E-mail: stuart-james.elliott@univ-lyon1.fr

${ }^{b}$ Institut de Chimie Radicalaire - UMR 7273, Saint-Jérôme Campus, Av. Esc. Normandie Niemen, Aix-Marseille Université/CNRS, 13397 Marseille, Cedex 20, France

${ }^{c}$ Bruker Switzerland, Industriestrasse 26, 8117 Fällanden, Switzerland

${ }^{d}$ Bruker Italia Srl, Viale Vincenzo Lancetti, 43, 20158 Milan, Italy

${ }^{e}$ Bruker Biospin, 15 Fortune Drive Billerica, Massachusetts, USA

$\dagger$ Electronic supplementary information (ESI) available. See DOI: 10.1039/ d1cp00429h
}

temperature is particularly slow, with low polarizations typically accrued over extensive timescales of hours or more. ${ }^{5}$ Shortened polarization build-up times are engendered by employing radiofrequency (rf) pulse sequences which efficiently transfer polarization from faster polarizing nuclear spins such as ${ }^{1} \mathrm{H} .{ }^{6}$

Cross-polarization (CP) boosts ${ }^{13} \mathrm{C}$ polarizations and shortens build-up time constants (by a factor of up to 40) under dDNP conditions (typ. at temperatures of $\sim 1.0-1.6 \mathrm{~K}$ in superfluid helium),${ }^{7-15}$ with a CP-DNP combination proving to be pivotal in the transportation of highly polarized metabolites. ${ }^{16}$ The CP method, however, is required to adhere to the strict Hartmann-Hahn matching condition, ${ }^{7}$ i.e. intense $B_{1}$-field matching (typ. $>15 \mathrm{kHz}$ ) of concurrent ${ }^{1} \mathrm{H}$ and ${ }^{13} \mathrm{C}$ spin-locking rf-irradiation during an optimized polarization transfer time (typ. $>1 \mathrm{~ms}$ ). This often leads to high rf-pulse powers for lowgamma nuclear spins and poor rf-pulse coverage of the NMR line in the case of the strongly polarized nuclear spins, i.e. the spinlocking performance of the high-gamma nuclear spins is strictly limited. The requirement of high power and energy rf-pulses risks detrimental arcing in the superfluid helium bath. This limitation has so far prevented the use of $\mathrm{CP}$ for sample volumes exceeding $500 \mu \mathrm{L}$, which is essential for the scaling-up of dDNP 
a) Zeeman Order

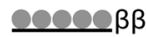
$a \beta$

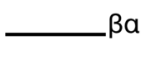

$\beta a$

ena $a$

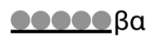

\section{eeneaa}

\section{enenaa}

Fig. 1 Pictorial representations of nuclear (a) Zeeman spin order $\left(\hat{I}_{z}\right)$ and (b) dipolar two-spin order $\left(\hat{l}_{i z} \cdot \hat{l}_{j z}\right)$. Black circles correspond to overpopulated energy levels, whilst grey circles correspond to underpopulated energy levels.

to sample volumes required for parallel hyperpolarization ${ }^{17}$ and human imaging. ${ }^{2-4} \mathrm{~A}$ minor side-effect is the heat introduced from the high-power rf-pulses, which in turn raises the temperature of the helium bath, and thus affects the DNP efficiency.

Polarization transfer between heteronuclear spins in the solid-state on static samples can alternatively be mediated in a "contactless" manner by a reservoir of dipolar order (see Fig. 1 for a representation of the dipolar order reservoir). ${ }^{18-28}$ Although rf-techniques for generating, evolving and reconverting dipolar order are demonstrated in the literature, little use has been documented since transfer efficiencies are dramatically affected in the case of magic angle spinning (MAS) NMR experiments $\ddagger$ However, there is no such limitation under the conditions of static DNP experiments at low temperatures. Furthermore, these alternative rf-methods advantageously often require lower power, and could therefore be more applicable for hyperpolarizing larger sample volumes.

In a previous Paper, ${ }^{29}$ we described the dynamics of a dipolar-order-mediated cross-polarization (dCP) rf-pulse sequence which relied on a contactless transfer of polarization from ${ }^{1} \mathrm{H}$ to ${ }^{13} \mathrm{C}$ nuclear spins hypothesized to be governed by a dipolar order reservoir under dDNP conditions. Polarization transfer via a bath of dipolar order was shown to perform well with respect to the rf-pulse powers employed, but the efficiency of the ${ }^{1} \mathrm{H} \rightarrow{ }^{13} \mathrm{C}$ polarization transfer process was found to be reduced with respect to a conventional $\mathrm{CP}$ approach for standard DNP-compatible sample formulations.

In the current Paper, we present an optimization of the dCP rf-pulse sequence for ${ }^{1} \mathrm{H}$ to ${ }^{13} \mathrm{C}$ polarization transfer in the context of dDNP experiments. We outline the following improvements: (i) extensions to shaped rf-pulses; and (ii) exploration of diversified spin systems with favourable molecular properties, such as stronger ${ }^{1} \mathrm{H}^{-13} \mathrm{C}$ dipolar couplings and absent $\mathrm{CH}_{3}$ group relaxation sinks. We report experimental observations for $\left[1-{ }^{13} \mathrm{C}\right]$ sodium acetate and other suitable molecules. We show improvements in the dCP transfer efficiency approaching factors of up to $\sim 1.65$ compared with previously reported results. ${ }^{29}$ ${ }^{1} \mathrm{H} \rightarrow{ }^{13} \mathrm{C}$ polarization transfer efficiencies of the dCP rf-pulse sequence on the order of $\sim 76 \%$ were realized with respect to a

‡ Gerd Buntkowsky, Personal Communication. conventional and optimized $\mathrm{CP}$ experiment, revealing ${ }^{13} \mathrm{C}$ polarizations in the region of $\sim 32.1 \%$ in the frozen solid after 10 minutes of ${ }^{1} \mathrm{H}$ DNP.

\section{Methods}

\subsection{Molecular systems}

The range of ${ }^{13} \mathrm{C}$-labelled molecular candidates under investigation in this study are shown in Table 1. Molecule $\mathbf{I}$ is used as a "benchmark" molecular system for which all rf-pulse sequences will be optimized. Molecules II and III are employed in order to verify the following hypotheses: (i) relocating the ${ }^{13} \mathrm{C}$ spin label to a carbon site adjacent to the methyl group protons will result in larger ${ }^{1} \mathrm{H}^{13} \mathrm{C}$ dipolar couplings which should strongly influence the ${ }^{1} \mathrm{H} \rightarrow{ }^{13} \mathrm{C}$ polarization transfer efficiency; and (ii) the absence of $\mathrm{CH}_{3}$ group relaxation sinks is expected to improve the dCP transfer efficiency, respectively.

\subsection{Sample preparation and freezing}

Solutions of $3 \mathrm{M}\left[1{ }^{-13} \mathrm{C}\right]$ sodium acetate $(\mathrm{I}),\left[2{ }^{-13} \mathrm{C}\right]$ sodium acetate (II) and $\left[1-{ }^{13} \mathrm{C}\right]$ sodium formate (III) in the glass-forming mixture $\mathrm{H}_{2} \mathrm{O} / \mathrm{D}_{2} \mathrm{O} /$ glycerol $-d_{8}(1 / 3 / 6 \mathrm{v} / \mathrm{v} / \mathrm{v})$ were doped with $50 \mathrm{mM}$ TEMPOL radical (all compounds purchased from Sigma Aldrich) and sonicated for $\sim 10$ minutes. Paramagnetic TEMPOL radicals (nitroxides) were chosen to most efficiently polarize ${ }^{1} \mathrm{H}$ spins under our dDNP conditions. $100 \mu \mathrm{L}$ volumes of each sample were separately pipetted into a Kel-F sample cup and inserted into a $7.05 \mathrm{~T}$ prototype Bruker Biospin polarizer equipped with a specialized dDNP probe and running TopSpin 3.5 software. The sample temperature was reduced to $1.2 \mathrm{~K}$ by submerging the sample in liquid helium and reducing the pressure of the variable temperature insert (VTI) towards $\sim 0.7$ mbar.

\subsection{Dynamic nuclear polarization and microwave gating}

The samples were polarized by applying microwave irradiation at $f_{\mu \mathrm{w}}=197.568 \mathrm{GHz}$ (positive lobe of the microwave spectrum) with triangular frequency modulation of amplitude $\Delta f_{\mu \mathrm{w}}=$ $\pm 120 \mathrm{MHz}^{30}$ and rate $f_{\text {mod }}=0.5 \mathrm{kHz}$ at a power of $c a . P_{\mu \mathrm{w}}=$ $125 \mathrm{~mW}$ at the output of the microwave source and $c a . P_{\mu \mathrm{w}}=30 \mathrm{~mW}$ reaching the DNP cavity, which were optimized by using sample $\mathbf{I}$ prior to commencing experiments to achieve the best possible level of ${ }^{1} \mathrm{H}$ polarization. Microwave gating was employed shortly before and during dDNP polarization transfer experiments to allow the electron spin ensemble to return to a highly polarized state, which

Table 1 Overview of the molecular candidates chosen for this study. Red: ${ }^{13} \mathrm{C}$ nuclear spins

\begin{tabular}{llll}
\hline Molecular name & $\begin{array}{l}{\left[1-^{13} \mathrm{C}\right] \text { Sodium }} \\
\text { acetate }\end{array}$ & $\begin{array}{l}{\left[2-{ }^{13} \mathrm{C}\right] \text { Sodium }} \\
\text { acetate }\end{array}$ & $\begin{array}{l}{\left[1-{ }^{13} \mathrm{C}\right] \text { Sodium }} \\
\text { formate }\end{array}$ \\
\hline Shorthand notation & $\mathrm{I}$ & $\mathrm{II}$ & $\mathrm{III}$ \\
Molecular structure & & &
\end{tabular}


happens on the timescale of the longitudinal electron relaxation time (typ. $T_{1 \mathrm{e}}=100 \mathrm{~ms}$ with $P_{\mathrm{e}}=99.93 \%$ under our conditions). ${ }^{31}$ Microwave gating hence provides a way to strongly attenuate paramagnetic relaxation, and consequently the ${ }^{1} \mathrm{H}$ and ${ }^{13} \mathrm{C} T_{1 \rho}$ relaxation time constants in the presence of spin-locking rf-fields are extended by orders of magnitude. This allows the spin-locking rf-pulses used to be much longer, which significantly increases the efficiency of nuclear polarization transfer.

\subsection{RF-Pulse sequences}

In the current study, we used variants of the adiabatic demagnetization/remagnetization in the rotating frame (ADRF/ARRF) rf-pulse sequence. ${ }^{20-28}$ The ADRF/ARRF rf-pulse sequence has been shown to possess improved dipolar order preparation and conversion efficiencies versus the non-Zeeman polarization transfer (NZPT) experiment proposed by Vieth and Yannoni. ${ }^{19}$ In our work, we will generally refer here to such polarization transfer schemes as dCP for dipolar-order-mediated crosspolarization. Fig. 2a shows this sequence adapted for our dDNP experiments.

The dCP rf-pulse sequence operates as follows:

(i) A crusher sequence of $90^{\circ}$ rf-pulses with alternating phases separated by a short delay (typ. $11 \mathrm{~ms}$ ) repeated $n$ times (typ. $n=50$ ) kills residual magnetization on both rf-channels;

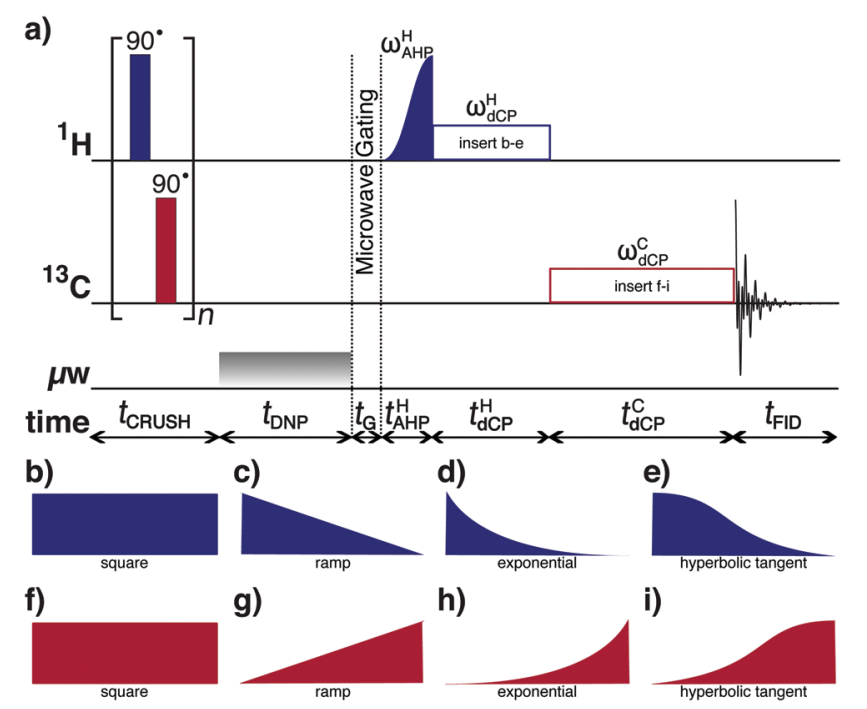

Fig. 2 (a) Schematic representation of the dCP rf-pulse sequence used for preparing ${ }^{1} \mathrm{H}-{ }^{1} \mathrm{H}$ dipolar order in I-III and monitoring its conversion to ${ }^{13} \mathrm{C}$ magnetization. The experiments used the following parameters chosen to maximize the efficiency of the dCP rf-pulse sequence: $n=$ $50 ; t_{\mathrm{G}}=0.5 \mathrm{~s} ; \omega_{\mathrm{AHP}}^{\mathrm{H}} / 2 \pi=27.8 \mathrm{kHz} ; t_{\mathrm{AHP}}^{\mathrm{H}}=175 \mu \mathrm{s}$. AHP = adiabatic halfpassage. AHP sweep width $=100 \mathrm{kHz}$. The $\pi / 2$ crusher rf-pulses use an empirically optimized thirteen-step phase cycle to remove residual magnetization at the beginning of each experiment: $\{0, \pi / 18,5 \pi / 18, \pi / 2$, $4 \pi / 9,5 \pi / 18,8 \pi / 9, \pi, 10 \pi / 9,13 \pi / 9, \pi / 18,5 \pi / 3,35 \pi / 18\}$. The resonance offset was placed at the centre of the ${ }^{1} \mathrm{H}$ and ${ }^{13} \mathrm{C}$ NMR peaks. $(b-e){ }^{1} \mathrm{H} d C P$ rf-pulse shapes implemented to prepare ${ }^{1} \mathrm{H}-{ }^{1} \mathrm{H}$ dipolar order in samples I-III. (f-i) ${ }^{13} \mathrm{C}$ dCP rf-pulse shapes employed to convert ${ }^{1} \mathrm{H}-{ }^{1} \mathrm{H}$ dipolar order to ${ }^{13} \mathrm{C}$ magnetization in samples I-III. All non-square rf-pulses have amplitudes ranging from $0 \% \rightarrow 100 \%\left({ }^{1} \mathrm{H}\right)$ or $100 \% \rightarrow 0 \%\left({ }^{13} \mathrm{C}\right)$. (ii) The microwave source becomes active for a time $t_{\mathrm{DNP}}$ during which ${ }^{1} \mathrm{H}$ DNP builds-up;

(iii) The microwave source is deactivated and a delay of duration $t_{\mathrm{G}}=0.5 \mathrm{~s}$ occurs before the next step, thus permitting the electron spins to relax to their highly polarized thermal equilibrium state; ${ }^{31}$

(iv) A preparatory ${ }^{1} \mathrm{H}$ adiabatic half-passage (AHP) rf-pulse creates transverse magnetization;

(v) A ${ }^{1} \mathrm{H}$ dCP rf-pulse of amplitude $\omega_{\mathrm{dCP}}^{\mathrm{H}}$ and length $t_{\mathrm{dCP}}^{\mathrm{H}}$ prepares ${ }^{1} \mathrm{H}-{ }^{1} \mathrm{H}$ dipolar order;

(vi) $\mathrm{A}^{13} \mathrm{C}$ dCP rf-pulse of amplitude $\omega_{\mathrm{dCP}}^{\mathrm{C}}$ and length $t_{\mathrm{dCP}}^{\mathrm{C}}$ presumably converts the ${ }^{1} \mathrm{H}-{ }^{1} \mathrm{H}$ dipolar order into ${ }^{13} \mathrm{C}$ magnetization;

(vii) The induced ${ }^{13} \mathrm{C}$ NMR signal is detected.

Further details regarding dCP rf-pulse sequence operation are given elsewhere. ${ }^{29}$

Fig. 2(b-i) indicates the ${ }^{1} \mathrm{H}$ and ${ }^{13} \mathrm{C}$ dCP rf-pulse shapes trialled to improve the performance efficiency of the dCP rf-pulse sequence. The amplitude $\omega_{\mathrm{dCP}}$ and duration $t_{\mathrm{dCP}}$ of the ${ }^{1} \mathrm{H}$ and ${ }^{13} \mathrm{C}$ dCP rf-pulse shapes were carefully optimized ${ }^{29}$ by detecting the induced NMR signal on the ${ }^{13} \mathrm{C}$ rf-channel in an iterative process until the intensity of the resulting ${ }^{13} \mathrm{C} N M R$ signals could not be improved further prior to each heteronuclear polarization transfer experiment.

\section{Results}

\subsection{Cross-polarization}

The performance efficiency of the dCP rf-pulse sequence was compared with a traditional CP experiment, ${ }^{7-15}$ which is described in the ESI, $\uparrow$ along with an rf-pulse sequence diagram, all optimized parameters and the ${ }^{13} \mathrm{C}$ polarization level $P_{\mathrm{CP}}\left({ }^{13} \mathrm{C}\right)$ achieved for each sample after a single CP contact. Experiments employed either 5 or $600 \mathrm{~s}$ of direct ${ }^{1} \mathrm{H}$ DNP at $1.2 \mathrm{~K}$ prior to polarization transfer to the ${ }^{13} \mathrm{C}$ heteronuclear spins. ${ }^{13} \mathrm{C}$ polarization levels in excess of $60 \%$ are anticipated by using a multiple CP contact approach. ${ }^{9-15}$ The ${ }^{13} \mathrm{C}$ polarization level $P_{\mathrm{dCP}}\left({ }^{13} \mathrm{C}\right)$ obtained as a result of using the dCP rf-pulse sequence is calculated by scaling $P_{\mathrm{CP}}\left({ }^{13} \mathrm{C}\right)$ by a factor of $I_{\mathrm{dCP}} /$ $I_{\mathrm{CP}}$, where $I_{\mathrm{dCP}}$ and $I_{\mathrm{CP}}$ are the integrals of the optimized dCP and $\mathrm{CP}{ }^{13} \mathrm{C}$ NMR signal maxima, respectively. $I_{\mathrm{dCP}} / I_{\mathrm{CP}}$ is referred to as the dCP rf-pulse sequence ${ }^{1} \mathrm{H} \rightarrow{ }^{13} \mathrm{C}$ nuclear polarization transfer efficiency.

\subsection{Shaped RF-pulses}

For all the samples employed in this study, it was found that the best performing ${ }^{1} \mathrm{H}$ and ${ }^{13} \mathrm{C}$ dCP rf-pulses (i.e. the most efficient rf-pulses for preparing and converting ${ }^{1} \mathrm{H}-{ }^{1} \mathrm{H}$ dipolar order) were a ramp which linearly decreases in amplitude (Fig. 2c) and an increasing amplitude hyperbolic tangent function (Fig. 2i), respectively. The amplitude $\omega_{\mathrm{dCP}}$ and duration $t_{\mathrm{dCP}}$ of the aforementioned ${ }^{1} \mathrm{H}$ and ${ }^{13} \mathrm{C}$ dCP rf-pulse shapes for the samples used in this investigation are shown in Table 2.

The experimental ${ }^{13} \mathrm{C}$ NMR signal intensities resulting from the above described shaped rf-pulse parameter optimization 
Table 2 Optimized dCP rf-pulse sequence parameters, peak rf-pulse powers $P_{\mathrm{dCP}}$ and peak rf-pulse energies $E_{\mathrm{dCP}}$ for the molecular derivatives used in this work protocol for sample I are detailed in Fig. 3. For the ${ }^{1} \mathrm{H}$ dCP rf-pulse it was found that the linearly decreasing amplitude ramp was a factor of $\sim 1.25$ more efficient in generating ${ }^{1} \mathrm{H}-{ }^{1} \mathrm{H}$ dipolar order than the practically simpler spin-locking ${ }^{1} \mathrm{H} \mathrm{dCP}$ rf-pulse (see Fig. 3a). In the case of the ${ }^{13} \mathrm{C}$ dCP rf-pulse, the hyperbolic tangent shaped rf-pulse was only a factor of $\sim 1.05$

a)

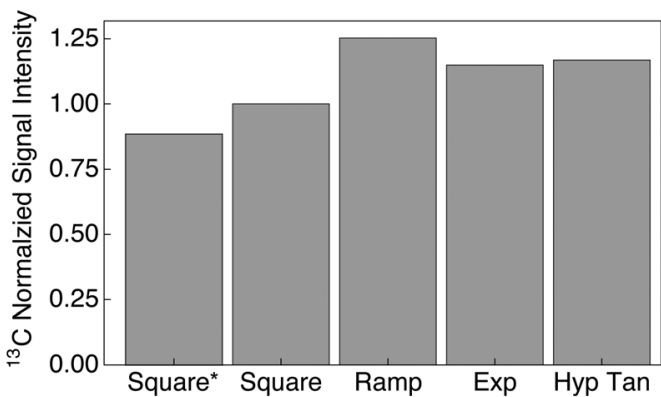

b)

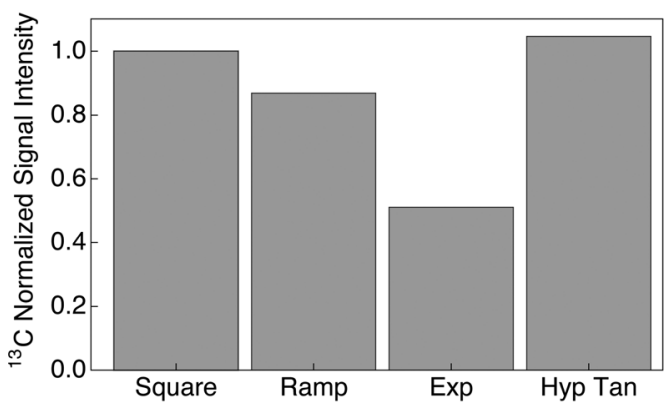

Fig. 3 Normalized ${ }^{13} \mathrm{C}$ NMR signal intensities of $\mathrm{I}$ as a function of the optimized (a) ${ }^{1} \mathrm{H}$ and (b) ${ }^{13} \mathrm{C}$ dCP rf-pulse shapes acquired at $7.05 \mathrm{~T}$ $\left({ }^{1} \mathrm{H}\right.$ nuclear Larmor frequency $=300.13 \mathrm{MHz},{ }^{13} \mathrm{C}$ nuclear Larmor frequency $=75.47 \mathrm{MHz}$ ) and $1.2 \mathrm{~K}$ with 1 transient after $5 \mathrm{~s}$ of ${ }^{1} \mathrm{H}$ DNP. ${ }^{*} \mathrm{AHP} \rightarrow$ hard $90^{\circ} \mathrm{rf}$-pulse (bandwidth $\simeq 40 \mathrm{kHz}$ ). Exp = Exponential; Hyp Tan = Hyperbolic Tangent. The ${ }^{13} \mathrm{C}$ NMR signal intensities in (a) were acquired by using the dCP rf-pulse sequence (Fig. 2a) with an increasing amplitude hyperbolic tangent ${ }^{13} \mathrm{C}$ dCP rf-pulse (Fig. 2i) of maximum amplitude $\omega_{\mathrm{d} C P}^{\mathrm{C}}$ and duration $t_{\mathrm{dCP}}^{\mathrm{C}}$ (Table 2). The ${ }^{13} \mathrm{C}$ NMR signal intensities in (b) were acquired by using the dCP rf-pulse sequence (Fig. 2a) with a linearly decreasing amplitude ramp ${ }^{1} \mathrm{H}$ dCP rf-pulse (Fig. 2C) of maximum amplitude $\omega_{\mathrm{dCP}}^{\mathrm{H}}$ and duration $t_{\mathrm{dCP}}^{\mathrm{H}}$ (Table 2). more efficient in supposedly transferring ${ }^{1} \mathrm{H}-{ }^{1} \mathrm{H}$ dipolar order to the ${ }^{13} \mathrm{C}$ heteronuclear spin (see Fig. 3b). It was also found that for all samples the effect of including a preparatory ${ }^{1} \mathrm{H} \mathrm{AHP}$ rf-pulse within the dCP rf-pulse sequence (in place of a hard $90^{\circ}$ rf-pulse) was to maximize the acquired ${ }^{1} \mathrm{H}$ NMR signal intensity, by a factor of $\sim 1.13$ (Fig. 3a) in the case of sample I, compared with the dCP rf-pulse sequence of Vieth and Yannoni. ${ }^{19,29}$ This leads to cumulative effect whereby the preparation efficiency of ${ }^{1} \mathrm{H}-{ }^{1} \mathrm{H}$ dipolar order is improved by a factor of $\sim 1.41$.

In comparison with a sophisticated CP approach (see Section 3.1 and the ESI $\dagger$ ) the integral of the optimized $\mathrm{dCP}^{13} \mathrm{C}$ NMR signal maximum for sample $\mathbf{I}$ is scaled by a factor of $\sim 0.46$ relative to the result achieved by using a high-power CP approach (see Table 3). This indicates a solid-state ${ }^{13} \mathrm{C}$ polarization of $P_{\mathrm{dCP}}\left({ }^{13} \mathrm{C}\right) \simeq 19.0 \%$. This is consistent with previous results reported in the literature. ${ }^{5,15}$ These results demonstrate that a dCP rf-pulse sequence containing shaped rf-pules has an increased efficiency for creating and transferring ${ }^{1} \mathrm{H}-{ }^{1} \mathrm{H}$ dipolar order.

\subsection{Molecular modifications}

Table 3 displays the ${ }^{1} \mathrm{H}$ polarization levels $P\left({ }^{1} \mathrm{H}\right),{ }^{13} \mathrm{C}$ nuclear polarizations obtained after a single ${ }^{1} \mathrm{H}-{ }^{1} \mathrm{H}$ dipolar order to ${ }^{13} \mathrm{C}$ magnetization conversion step $P_{\mathrm{dCP}}\left({ }^{13} \mathrm{C}\right)$ and ${ }^{1} \mathrm{H} \rightarrow{ }^{13} \mathrm{C}$ nuclear polarization transfer efficiencies $I_{\mathrm{dCP}}\left({ }^{13} \mathrm{C}\right) / I_{\mathrm{CP}}\left({ }^{13} \mathrm{C}\right)$ for each molecular candidate in our series of experiments. The value of $I_{\mathrm{dCP}}\left({ }^{13} \mathrm{C}\right) / I_{\mathrm{CP}}\left({ }^{13} \mathrm{C}\right)$ was determined using just $5 \mathrm{~s}$ of ${ }^{1} \mathrm{H} \mathrm{DNP}$.

All samples show a similar level of ${ }^{1} \mathrm{H}$ polarization after $5 \mathrm{~s}$ of ${ }^{1} \mathrm{H}$ DNP, however, the ${ }^{13} \mathrm{C}$ polarizations can significantly differ. The ${ }^{1} \mathrm{H} \rightarrow{ }^{13} \mathrm{C}$ nuclear polarization transfer efficiencies follow a similar trend. It is worth noting that, after 10 minutes of ${ }^{1} \mathrm{H}$ DNP and a sole dCP nuclear polarization transfer step, sample II achieves an impressive ${ }^{13} \mathrm{C}$ polarization of $\sim 32.1 \%$ (with a conversion efficiency of $\sim 76 \%$ for the case of $t_{\mathrm{DNP}}=5 \mathrm{~s}$ ). These results indicate that although the protons within each sample polarize to a similar extent, the ${ }^{13} \mathrm{C}$ polarization of each sample is dominated by the efficiency of the nuclear polarization transfer stages in the dCP rf-pulse sequence. This trend also appears to correlate with the nature and number of bonded ${ }^{1} \mathrm{H}$ nuclear spins.

Table $3{ }^{1} \mathrm{H}$ and ${ }^{13} \mathrm{C}$ nuclear spin polarization levels $P\left({ }^{1} \mathrm{H}\right)$ and $P_{\mathrm{dCP}}\left({ }^{13} \mathrm{C}\right)$, respectively, and dCP rf-pulse sequence ${ }^{1} \mathrm{H} \rightarrow{ }^{13} \mathrm{C}$ nuclear polarization transfer efficiencies $I_{\mathrm{dCP}}\left({ }^{13} \mathrm{C}\right) / I_{\mathrm{CP}}\left({ }^{13} \mathrm{C}\right)$ for the molecular derivatives used in this work

\begin{tabular}{|c|c|c|c|c|}
\hline \multirow[b]{3}{*}{ Quantity } & & \multicolumn{3}{|c|}{ Molecules } \\
\hline & & I & II & III \\
\hline & $t_{\mathrm{DNP}}$ & $\mathrm{H}_{3} \mathrm{C}^{\prime}$ & $\mathrm{H}_{3}{ }^{13} \mathrm{C}^{-}$ & \\
\hline$P\left({ }^{1} \mathrm{H}\right) / \%$ & $5 \mathrm{~s}$ & 4.4 & 4.2 & 4.7 \\
\hline$P_{\mathrm{dCP}}\left({ }^{13} \mathrm{C}\right) / \%$ & $5 \mathrm{~s}$ & 1.4 & 2.7 & 1.6 \\
\hline$P_{\mathrm{dCP}}\left({ }^{13} \mathrm{C}\right) / \%$ & $600 \mathrm{~s}$ & 19.0 & 32.1 & - \\
\hline$I_{\mathrm{dCP}}\left({ }^{13} \mathrm{C}\right) / I_{\mathrm{CP}}\left({ }^{13} \mathrm{C}\right) / \%$ & $5 \mathrm{~s}$ & 45.8 & 76.0 & 57.2 \\
\hline
\end{tabular}


Table $4{ }^{1} \mathrm{H}$ molarities $\mathrm{C}\left({ }^{1} \mathrm{H}\right),{ }^{13} \mathrm{C}$ dCP rf-pulse build-up rate constants $\left(R_{\mathrm{dCP}}^{C}=1 / \tau_{\mathrm{dCP}}^{C}\right)$ and effective $\mathrm{dCP}$ build-up rate constants $R_{\mathrm{dCP}}^{*}$ for the samples investigated during this work (see text for details)

\begin{tabular}{|c|c|c|c|c|}
\hline \multirow[b]{3}{*}{ Quantity } & \multirow[b]{3}{*}{ Units } & \multicolumn{3}{|l|}{ Molecules } \\
\hline & & I & II & III \\
\hline & & $\mathrm{H}_{3} \mathrm{C}^{\prime}$ & ${ }^{8} \mathrm{C}^{-}$ & \\
\hline$C\left({ }^{1} \mathrm{H}\right)$ & & 20.07 & 20.07 & 14.07 \\
\hline$R_{\mathrm{dCP}}^{\mathrm{C}} \times 10^{3}$ & $\mathrm{~ms}^{-1}$ & $69.9 \pm 1.0$ & $59.5 \pm 0.7$ & $40.3 \pm 3.6$ \\
\hline$R_{\mathrm{dCP}}^{*} \times 10^{3}$ & $\mathrm{M}^{-1} \mathrm{~ms}^{-1}$ & $3.48 \pm 0.05$ & $2.97 \pm 0.04$ & $2.9 \pm 0.3$ \\
\hline
\end{tabular}

Table 4 indicates the proton molarities $C\left({ }^{1} \mathrm{H}\right),{ }^{13} \mathrm{C} \mathrm{dCP}$ rf-pulse build-up rate constants $\left(R_{\mathrm{dCP}}^{\mathrm{C}}=1 / \tau_{\mathrm{dCP}}^{\mathrm{C}}\right)$ and effective dCP build-up rate constants $R_{\mathrm{dCP}}^{*}$ for the samples employed in our experiments under dDNP conditions, where the effective dCP build-up rate constant $R_{\mathrm{dCP}}^{*}$ is defined as:

$$
R_{\mathrm{dCP}}^{*}=\frac{R_{\mathrm{dCP}}^{\mathrm{C}}}{C\left({ }^{1} \mathrm{H}\right)}
$$

i.e. the ${ }^{13} \mathrm{C} \mathrm{dCP}$ rf-pulse build-up rate constant per ${ }^{1} \mathrm{H}$ sample molarity.

The ${ }^{13} \mathrm{C}$ dCP rf-pulse build-up rate constants $R_{\mathrm{dCP}}^{\mathrm{C}}$ were extracted by following the presumed conversion of ${ }^{1} \mathrm{H}-{ }^{1} \mathrm{H}$ dipolar order to ${ }^{13} \mathrm{C}$ magnetization using the dCP rf-pulse sequence described in Fig. 2, and the associated text, with $t_{\mathrm{DNP}}=5 \mathrm{~s}$. The experimental ${ }^{13} \mathrm{C}$ polarization build-up curves (shown in the ESI $\dagger$ ) are well fitted with a stretched exponential function using a build-up rate constant denoted $R_{\mathrm{dCP}}^{\prime}$. Stretched exponential build-up function: $A\left(1-\exp \left\{-\left(R_{\mathrm{dCP}}^{\prime} t\right)^{\beta}\right\}\right)$, where $A$ is a constant, $R_{\mathrm{dCP}}^{\prime}$ is the ${ }^{13} \mathrm{C}$ dCP rf-pulse build-up rate constant extracted from the above fitting procedure and $\beta$ is the breadth of the distribution of ${ }^{13} \mathrm{C} \mathrm{dCP}$ rf-pulse build-up rate constants. The average ${ }^{13} \mathrm{C}$ dCP rf-pulse build-up rate constants $R_{\mathrm{dCP}}^{\mathrm{C}}$ are calculated as follows: $R_{\mathrm{dCP}}^{\mathrm{C}}=R_{\mathrm{dCP}}^{\prime} \beta / \Gamma(1 / \beta)$, where $\Gamma(1 / \beta)$ is the gamma function.

The ${ }^{13} \mathrm{C}$ dCP rf-pulse build-up rate constant $R_{\mathrm{dCP}}^{\mathrm{C}}$ for samples without methyl groups, i.e. sample III, was found to be reduced. However, the effective dCP build-up rate constants $\left(R_{\mathrm{dCP}}^{*}\right)$ for all samples used in this study are in approximate agreement.

The lifetime of dipolar order for samples I and III was measured by using the variant of the dCP rf-pulse sequence depicted in Fig. 4a. The dCP rf-pulse sequence is repeated with incremented values of the variable evolution delay $\left(\tau_{\mathrm{EV}}\right)$ to monitor the decay of dipolar order. By fitting the integrated ${ }^{13} \mathrm{C}$ NMR signal decay as a function of the variable evolution time $\left(\tau_{\mathrm{EV}}\right)$ the dipolar order lifetime can be estimated.

Experimental decay curves showing the relaxation of dipolar order are presented in Fig. 4b. The decay of dipolar order was found to have a stretched exponential behaviour in both cases. The experimental decays are well fitted with a stretched exponential decay function (solid lines) using a relaxation time constant denoted $T_{z z}^{\prime}$. Stretched exponential decay function: $A \exp \left\{-\left(t / T_{z z}^{\prime}\right)^{\beta}\right\}$, where $A$ is a constant, $T_{z z}^{\prime}$ is the dipolar order a)

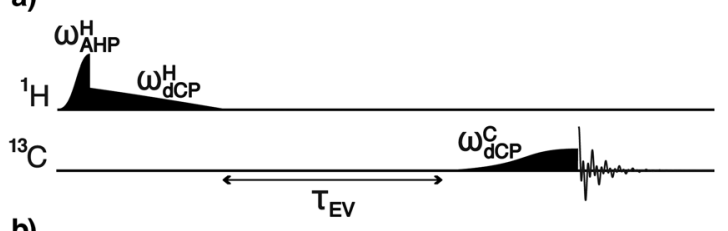

b)

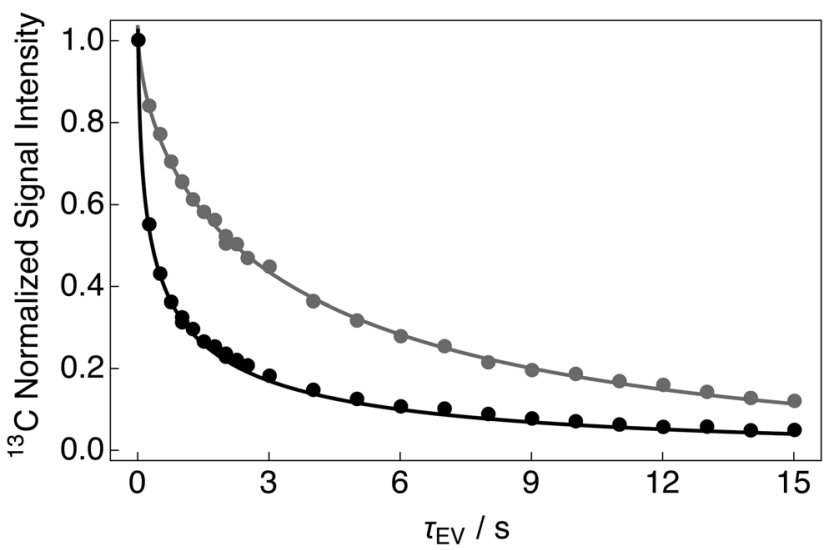

Fig. 4 (a) Schematic representation of the variant of the dCP rf-pulse sequence used for preparing dipolar order and monitoring its decay. (b) Experimental relaxation curves showing the decay of dipolar order for samples I (black) and III (grey) acquired at $7.05 \mathrm{~T}\left({ }^{1} \mathrm{H}\right.$ nuclear Larmor frequency $=300.13 \mathrm{MHz},{ }^{13} \mathrm{C}$ nuclear Larmor frequency $=75.47 \mathrm{MHz}$ ) and $1.2 \mathrm{~K}$ with a single transient per data point. All signal amplitudes were normalized to the first data point. The decay curves were fitted with a stretched exponential decay function: $A \exp \left\{\left(-t / T_{z z}^{\prime}\right)^{\beta}\right\}$. Mean dipolar order lifetimes $\left(T_{z z}=T_{z z}^{\prime} \Gamma(1 / \beta) / \beta\right)$ : Black curve: $T_{z z}=2.3 \pm 0.2 \mathrm{~s}$; Grey curve: $T_{z z}=$ $6.0 \pm 0.1 \mathrm{~s}$ (see text for details).

relaxation time constant extracted from the above fitting procedure and $\beta$ is the breadth of the distribution of dipolar order relaxation time constants. The mean dipolar order relaxation time constants $T_{\mathrm{zz}}$ are calculated as follows: $T_{z z}=$ $T_{z z}^{\prime} \Gamma(1 / \beta) / \beta$, where $\Gamma(1 / \beta)$ is the gamma function. In these cases, the measured average lifetimes of dipolar order $T_{z z}$ are: Sample I: $T_{z z}=2.3 \pm 0.2 \mathrm{~s}$; and Sample III: $T_{z z}=6.0 \pm 0.1 \mathrm{~s}$.

\section{Discussion}

\subsection{Shaped RF-pulses}

In prior work, ${ }^{29}$ we investigated the spin dynamics of ${ }^{1} \mathrm{H} \rightarrow{ }^{13} \mathrm{C}$ nuclear polarization transfer by employing a dCP rf-pulse sequence which uses sequential spin-locking ${ }^{1} \mathrm{H}$ and ${ }^{13} \mathrm{C} \mathrm{dCP}$ rf-pulses. The polarization transfer efficiency of the dCP rf-pulse sequence was found to be reasonable with respect to the conversion performance of a sophisticated and high-power cross-polarization experiment given the dCP rf-pulse powers employed. We postulated above that the dCP rf-pulse sequence performance efficiency could be increased further by the implementation and optimization of shaped rf-pulses.

A number of non-constant amplitude rf-pulses have previously been reported in the literature in the context of heteronuclear polarization transfer via a reservoir of ${ }^{1} \mathrm{H}^{-}{ }^{1} \mathrm{H}$ dipolar order, and examples include the ADRF/ARRF rf-pulse sequence. ${ }^{20-28}$ Such 
rf-pulse sequences are known to be efficient with respect to the transfer of polarization between heteronuclear spins but are more difficult to implement technically compared with the more conventional dCP rf-pulse sequence. ${ }^{19,29}$

Non-constant amplitude ${ }^{1} \mathrm{H}$ dCP rf-pulses slowly transform transverse ${ }^{1} \mathrm{H}$ magnetization into ${ }^{1} \mathrm{H}-{ }^{1} \mathrm{H}$ dipolar order, and often require longer rf-pulse durations compared with spinlocking dCP rf-pulses. Shaped rf-pulses also allow finer control over the amplitude $\omega_{\mathrm{dCP}}^{\mathrm{H}}$, and span a number of different $B_{1}$-field strengths, which appears more beneficial for the preparation of ${ }^{1} \mathrm{H}-{ }^{1} \mathrm{H}$ dipolar order. As a result of the improved dCP rf-pulse sequence adiabaticity, it is possible that proton dipolar order is generated in larger quantities. The results demonstrated in Fig. 3 confirm this phenomenon $\left({ }^{1} \mathrm{H}\right.$ dCP rf-pulse durations, after optimization, are approximately one order of magnitude longer for shaped rf-pulses compared with spin-locking rf-pulses ${ }^{29}$ ) and indicate that, in suitable circumstances, the polarization transfer performance of the entire dCP rf-pulse sequence is most likely to be governed by the preparation efficiency of ${ }^{1} \mathrm{H}-{ }^{1} \mathrm{H}$ dipolar order (see Fig. 3a), since the improved shape of the ${ }^{13} \mathrm{C}$ dCP rf-pulse has relatively little influence on the resulting ${ }^{13} \mathrm{C}$ NMR signal integral (see Fig. 3b).

Furthermore, the inclusion of an adiabatic half-passage (AHP) additionally improved the transfer efficiency of the dCP rf-pulse sequence. AHP rf-pulses are well-known to: (i) achieve broadband excitation which is insensitive to spatially inhomogeneous magnetic fields (such as those present in our dDNP apparatus); ${ }^{32}$ and (ii) prepare non-negligible quantities of dipolar order. ${ }^{33}$ This improvement in efficiency could be due to either the additional broadband character of the ${ }^{1} \mathrm{H}$ AHP rf-pulse excitation profile or the creation of surplus ${ }^{1} \mathrm{H}-{ }^{1} \mathrm{H}$ dipolar order. We did not investigate the effects of generating ${ }^{1} \mathrm{H}-{ }^{1} \mathrm{H}$ dipolar order by using ${ }^{1} \mathrm{H}$ AHP rf-pulses further.

\section{2. ${ }^{13} \mathrm{C}$-Labelling strategies}

Sample $\mathbf{I}$ is a typical molecule of choice for dDNP experiments, due its biochemical importance and long spin-lattice relaxation time constants in solution. Sample II has recently been suggested as a novel lineshape polarimeter, ${ }^{34}$ and the location of ${ }^{13} \mathrm{C}$ spin labels has previously been explored with respect to dDNP performances. ${ }^{35}$

There is a major improvement in the observed dCP sequence transfer efficiency and final ${ }^{13} \mathrm{C}$ polarization for sample II compared with sample I (see Table 3). This is likely associated with the fact that sample II has three directly-bound ${ }^{1} \mathrm{H}$ nuclear spins, whereas the ${ }^{1} \mathrm{H}$ nuclear spins in sample I are not directly bonded. The larger ${ }^{1} \mathrm{H}_{-}{ }^{13} \mathrm{C}$ dipolar coupling constants likely lead to an improved dCP rf-pulse sequence performance efficiency, given the similar initial ${ }^{1} \mathrm{H}$ polarizations (see Table 3 ).

During the ${ }^{13} \mathrm{C}$ dCP rf-pulse, the ${ }^{13} \mathrm{C}$ polarization build-up rate constants $R_{\mathrm{dCP}}^{\mathrm{C}}$ for the samples employed in this study can also be used to infer information regarding the key factors of the supposed ${ }^{1} \mathrm{H}-{ }^{1} \mathrm{H}$ dipolar order to ${ }^{13} \mathrm{C}$ magnetization conversion process. It is worth noting that in the cases of samples I and II, the value of $R_{\mathrm{dCP}}^{\mathrm{C}}$ appears to be approximately independent of the molecular labelling position of the ${ }^{13} \mathrm{C}$ site. This is an unexpected result, given the increased ${ }^{1} \mathrm{H}-{ }^{13} \mathrm{C}$ dipolar coupling constants in sample II. The ${ }^{13} \mathrm{C}$ dCP rf-pulse build-up rate constant for sample II is therefore longer compared with our expectations, implying a slower than anticipated ${ }^{1} \mathrm{H}-{ }^{1} \mathrm{H}$ dipolar order to ${ }^{13} \mathrm{C}$ polarization transfer.

At present, we do not have a clear explanation of the above described results. The proximity of the ${ }^{13} \mathrm{C}$-labelled nuclear site of interest to the molecularly bound ${ }^{1} \mathrm{H}$ nuclear spins, and any resulting deleterious relaxation phenomena, may be responsible, and as such; this result could be related to the presence of the methyl group in samples I and II. These influences will be described in the next section.

It is of interest to compare the ${ }^{13} \mathrm{C}$ polarization accrued per unit time for the $\mathrm{dCP}$ method with direct ${ }^{13} \mathrm{C}$ DNP. After $\sim 30$ minutes of directly polarizing ${ }^{13} \mathrm{C}$ nuclear spins, ${ }^{13} \mathrm{C}$ polarizations on the order of $c a$. $\sim 10-12 \%$ are typically achieved. ${ }^{10,12}$ This gives a ${ }^{13} \mathrm{C}$ polarization per unit time of $\sim 0.33-0.4 \% \mathrm{~min}^{-1}$. For the dCP approach, the ${ }^{13} \mathrm{C}$ polarization per unit time for the case of sample II is $\sim 3.2 \% \mathrm{~min}^{-1}$. This corresponds to an improvement in the level of ${ }^{13} \mathrm{C}$ polarization by a factor of $\sim 8.0-9.7$ coupled with a three-fold acceleration of the ${ }^{13} \mathrm{C}$ polarization build-up rate constant, compared with the case of direct ${ }^{13} \mathrm{C}$ DNP. As shown in the ESI, $\dagger$ improved results may also be achieved by implementing state-of-the-art and optimized CP rf-pulse sequences. Future dDNP experiments should take advantage of the described dCP and CP methodologies, and their multiple-step variants, ${ }^{12,31}$ if the experimental apparatus permits the implementation of such rf-pulse techniques.

\subsection{Removal of methyl groups}

The rapid rotational motion of methyl group moieties can act as strong relaxation sinks for nearby magnetic nuclear spins. The rotation of $\mathrm{CH}_{3}$ groups might remain active even at liquid helium temperatures. ${ }^{36}$ As such, it is possible that the methyl group of I can cause significant nuclear spin relaxation of ${ }^{1} \mathrm{H}-{ }^{1} \mathrm{H}$ dipolar order. In an effort to combat this detrimental relaxation phenomenon we investigated sample III, which lacks a methyl group moiety. Sample III is a common molecular target in zero and ultra-low field (ZULF) NMR experiments, and the combination of $\mathrm{dDNP}$ and ZULF NMR is very promising.

The dCP rf-pulse sequence is more efficient for sample III than sample I under the same experimental conditions (see Table 3). This is likely to again be related to both the nature and number of the molecularly bound ${ }^{1} \mathrm{H}$ nuclear spins. Although sample I has three attached protons, these ${ }^{1} \mathrm{H}$ spins are somewhat distant to the ${ }^{13} \mathrm{C}$-labelled nuclear site, and hence the ${ }^{1} \mathrm{H}-{ }^{13} \mathrm{C}$ dipolar couplings are relatively weak. The ${ }^{1} \mathrm{H}-{ }^{13} \mathrm{C}$ dipolar coupling in sample III is much stronger due to the direct bonding nature of the spin constituents. The reduced number of directly bound ${ }^{1} \mathrm{H}$ spins in this system also possibly explains the dCP rf-pulse sequence efficiency with respect to that of sample II. These results, and those discussed in Section 4.2, demonstrate that the presence of multiple directly bound ${ }^{1} \mathrm{H}$ spins, and hence the number and strength of ${ }^{1} \mathrm{H}-{ }^{13} \mathrm{C}$ dipolar 
couplings, is advantageous for improving the dCP polarization transfer mechanism.

This result also indicates that the build-up of ${ }^{1} \mathrm{H}-{ }^{1} \mathrm{H}$ dipolar order, and presumed subsequent conversion to ${ }^{13} \mathrm{C}$ magnetization, can tolerate (although with a loss of ${ }^{13} \mathrm{C}$ NMR signal intensity) the influence of $\mathrm{CH}_{3}$ group relaxation sinks (possibly due to the sufficiently reduced presence of methyl group rotation at $1.2 \mathrm{~K}$ ). This statement is qualified by comparing the ${ }^{13} \mathrm{C}$ dCP rf-pulse build-up rate constant $R_{\mathrm{dCP}}^{\mathrm{C}}$ for samples I and III, which is only a factor of $\sim 1.73$ smaller than for sample III given the type of different molecular moieties near the ${ }^{13} \mathrm{C}$-labelled sites (see Table 4).

Although the ${ }^{13} \mathrm{C}$ polarization for III was not recorded after 10 minutes of ${ }^{1} \mathrm{H}$ DNP, the relatively favourable efficiency of the dCP rf-pulse sequence for this sample indicates that $P_{\mathrm{dCP}}\left({ }^{13} \mathrm{C}\right)$ would have exceeded $\sim 20 \%$ under our experimental dDNP conditions (see Table 3).

As demonstrated in Fig. 4b, the mean lifetime of dipolar order $T_{z z}$ for sample I is shorter than that for sample III. This result is attributed to the presence of the methyl group of $\left[1-{ }^{13} \mathrm{C}\right]$ sodium acetate, which acts as a relaxation sink for dipolar order established between pairs of proton spins in the sample. However, dipolar order lifetimes can span over many orders of magnitude between different samples. ${ }^{37}$ This is illustrated in Fig. 4b where dipolar order relaxation slows down by a factor of $\sim 2.6$ upon removal of the methyl moiety in sample I.

Furthermore, the stretched mono-exponential nature of the dipolar order decay is not fully understood but is potentially thought to be related to interactions with nearby ${ }^{1} \mathrm{H}$ spins in the DNP solvent. Such protons are located at a distribution of distances, and hence would explain why a stretched exponential function best fits the experimental data. The presented dipolar order lifetimes are typically shorter by 3 to 4 orders of magnitude than those of ${ }^{1} \mathrm{H}$ polarization (typically more than an hour) under the same experimental conditions. ${ }^{38}$

\subsection{RF-Powers and energies}

The rf-power requirements for polarization transfer are dependent upon the rf-pulse sequence used, the molecular spin system of interest, the capabilities of the dDNP probe and potentially the choice of application. Table 2 shows the peak rf-pulse powers $P_{\mathrm{dCP}}$ and energies $E_{\mathrm{dCP}}$ required for heteronuclear polarization transfer via the dCP rf-pulse sequence under dDNP conditions for each molecular candidate employed in our series of experiments. The dCP rf-pulse sequence powers and energies used were compared to those of an optimized state-of-the-art CP experiment for each sample (see the ESI, $\dagger$ for more details). ${ }^{7-15}$

In general, the peak power for the ${ }^{13} \mathrm{C} \mathrm{dCP}$ rf-pulse is up to $\sim 3.8$ times lower than required for $\mathrm{CP}$ (see Table 2). The considerably lower peak ${ }^{13} \mathrm{C}$ dCP rf-pulse power is exceedingly beneficial. Not only is there a significantly reduced tendency for detrimental rf-probe arcing incidents within the superfluid liquid helium bath but the decreased peak rf-pulse power requirement also ensures stable rf-pulse amplitudes at adequately long rf-pulse durations, which is highly advantageous in the case that the applied $B_{1}$-field of the rf-probe is weak or unstable at higher peak rf-pulse powers. Furthermore, since the total rf-energy deposited is reduced, the temperature variation of the liquid helium reservoir is likewise decreased, and the thermal shock applied to the cold and rigid capacitors is much smaller, which extends the lifetime of rf-probe circuitry, and hence rf-probe functionality, in such a far from standard environment.

The longer durations of the shaped ${ }^{1} \mathrm{H}$ dCP rf-pulses allow for the implementation of reduced rf-pulse amplitudes with respect to a conventional spin-locking rf-pulse, ${ }^{29}$ in suitable cases, and as a result; a comparison with $\mathrm{CP}$ provides an immediate benefit in terms of the deposited rf-energies. The peak ${ }^{1} \mathrm{H}$ rf-powers of both rf-pulse sequences are the same order of magnitude, but the duration of the ${ }^{1} \mathrm{H}$ dCP rf-pulse is easily a factor of $\sim 12.6-60.0$ times shorter than utilized for sufficient CP.

Furthermore, since the CP rf-pulse sequence is only operational under the application of simultaneous rf-pulses, and since the peak rf-pulse powers for each rf-pulse sequence are mostly governed by the ${ }^{13} \mathrm{C}$ rf-pulses, the peak rf-pulse power for the entire dCP rf-pulse sequence is significantly lower than that of $\mathrm{CP}$, which is the main rf-pulse sequence characteristic responsible for rf-probe arcing.

Although the deposited rf-energies for shaped ${ }^{1} \mathrm{H} \mathrm{dCP}$ rf-pulses are greater (due to extended ${ }^{1} \mathrm{H}$ rf-pulse durations) compared with conventional spin-locking rf-pulses, ${ }^{29}$ this result is outweighed by the increased generation of ${ }^{1} \mathrm{H}-{ }^{1} \mathrm{H}$ dipolar order and hence an improved performance efficiency of the dCP rf-pulse sequence compared to optimized CP.

Consequently, the reduced deposited ${ }^{1} \mathrm{H}$ and ${ }^{13} \mathrm{C}$ peak rf-powers required for a successful implementation of the dCP rf-pulse sequence provide a suitable alternative polarization transfer approach to $\mathrm{CP}$, with the less strict optimization of the $\mathrm{dCP}$ rf-pulse sequence a strong benefit for potential applications in a clinical environment.

\section{Conclusions}

We have demonstrated that it is possible to improve ${ }^{1} \mathrm{H} \rightarrow{ }^{13} \mathrm{C}$ polarization transfer techniques which operate by passing nuclear spin order through a bath of ${ }^{1} \mathrm{H}-{ }^{1} \mathrm{H}$ dipolar terms. The increased performance of the dCP rf-pulse sequence was achieved by adhering to the following protocols: (i) implementing shaped rf-pulses; (ii) employing suitable ${ }^{13} \mathrm{C}$ spin labelling modifications; and (iii) utilizing molecular derivatives which lack a methyl group moiety. Enhancements of ${ }^{13} \mathrm{C}$ NMR signal intensities were observed in all cases, with improvements approaching factors of $\sim 1.65$ compared with previously reported results. $^{29}$ The dCP rf-pulse sequence obtained ${ }^{1} \mathrm{H} \rightarrow{ }^{13} \mathrm{C}$ polarization transfer efficiencies approaching $\sim 76 \%$ compared with the performance of a high-power and energy CP experiment. ${ }^{13} \mathrm{C}$ polarization levels up to $\sim 32.1 \%$ were achieved by using the dCP rf-pulse sequence after 10 minutes of ${ }^{1} \mathrm{H}$ DNP.

The results of our experimental study indicate that ${ }^{13} \mathrm{C}$ nuclear spins at high concentrations can be readily polarized 
by a simple and low power rf-pulse sequence which requires minimal rf-pulse optimization. The findings also suggest that the dCP rf-pulse sequence requires a number of proximal ${ }^{1} \mathrm{H}$ spins in order to operate to a high polarization transfer efficiency. This effect is clearly highlighted for the case of sample II. This phenomenon is explored in more detail in a future publication. ${ }^{39}$ These observations are encouraging for the future usage of the dCP rf-pulse sequence, permitting hyperpolarization experiments conducive to larger sample volumes such as those compatible with clinical studies performed on human patients.

\section{Conflicts of interest}

There are no conflicts to declare.

\section{Acknowledgements}

This research was supported by ENS-Lyon, the French CNRS, Lyon 1 University, the European Research Council under the European Union's Horizon 2020 research and innovation program (ERC Grant Agreements No. 714519/HP4 all and Marie Skłodowska-Curie Grant Agreement No. 766402/ZULF). The authors gratefully acknowledge Bruker Biospin for providing the prototype dDNP polarizer, and particularly Marc Rossire and Marco Sacher for scientific and technical support. The authors additionally acknowledge Gerd Buntkowsky (Technische Universitat Darmstadt) who kindly communicated data associated with prior publications to us; Burkhard Luy (Karlsruhe Institute of Technology) for enlightening discussions; Catherine Jose and Christophe Pages for use of the ISA Prototype Service; Stéphane Martinez of the UCBL mechanical workshop for machining parts of the experimental apparatus; and the reviewers for their comments which led to the improvement of this article.

\section{References}

1 J. H. Ardenkjær-Larsen, B. Fridlund, A. Gram, G. Hansson, L. Hansson, M. H. Lerche, R. Servin, M. Thaning and K. Golman, Proc. Natl. Acad. Sci. U. S. A., 2003, 100, 10158-10163.

2 S. E. Day, M. I. Kettunen, F. A. Gallagher, D.-E. Hu, M. Lerche, J. Wolber, K. Golman, J. H. Ardenkjær-Larsen and K. M. Brindle, Nat. Med., 2007, 13, 1382-1387.

3 K. M. Brindle, S. E. Bohndiek, F. A. Gallagher and M. I. Kettunen, Magn. Reson. Med., 2011, 66, 505-519.

4 S. J. Nelson, J. Kurhanewicz, D. B. Vigneron, P. E. Z. Larson, A. L. Harzstark, M. Ferrone, M. van Criekinge, J. W. Chang, R. Bok, I. Park, G. Reed, L. Carvajal, E. J. Small, P. Munster, V. K. Weinberg, J. H. Ardenkjær-Larsen, A. P. Chen, R. E. Hurd, L.-I. Odegardstuen, F. J. Robb, J. Tropp and J. A. Murray, Sci. Transl. Med., 2013, 5, 198.
5 J. M. O. Vinther, V. Zhurbenko, M. M. Albannay and J. H. Ardenkjær-Larsen, Solid State Nucl. Mag., 2019, 102, 12-20.

6 G. Hartmann, D. Hubert, S. Mango, C. C. Morehouse and K. Plog, Nucl. Instrum. Methods Phys. Res., Sect. A, 1973, 106, 9-12.

7 S. R. Hartmann and E. L. Hahn, Phys. Rev., 1962, 128, 204-205.

8 A. Pines, M. Gibby and J. Waugh, Chem. Phys. Lett., 1972, 15, 373-376.

9 S. Jannin, A. Bornet, S. Colombo and G. Bodenhausen, Chem. Phys. Lett., 2011, 517, 234-236.

10 A. Bornet, R. Melzi, S. Jannin and G. Bodenhausen, Appl. Magn. Reson., 2012, 43, 107-117.

11 M. Batel, M. Krajewski, A. Däpp, A. Hunkeler, B. H. Meier, S. Kozerke and M. Ernst, Chem. Phys. Lett., 2012, 554, 72-76.

12 A. Bornet, R. Melzi, A. J. Perez Linde, P. Hautle, B. van den Brandt, S. Jannin and G. Bodenhausen, J. Chem. Phys. Lett., 2013, 4, 111-114.

13 B. Vuichoud, A. Bornet, F. de Nanteuil, J. Milani, E. Canet, X. Ji, P. Miéville, E. Weber, D. Kurzbach, A. Flamm, R. Konrat, A. D. Gossert, S. Jannin and G. Bodenhausen, Chem. - Eur. J., 2016, 22, 14696-14700.

14 M. Cavaillès, A. Bornet, X. Jaurand, B. Vuichoud, D. Baudouin, M. Baudin, L. Veyre, G. Bodenhausen, J.-N. Dumez, S. Jannin, C. Copéret and C. Thieuleux, Angew. Chem., Int. Ed., 2018, 130, 7575-7579.

15 A. J. Perez Linde, PhD thesis, University of Nottingham, UK, 2009.

16 X. Ji, A. Bornet, B. Vuichoud, J. Milani, D. Gajan, A. J. Rossini, L. Emsley, G. Bodenhausen and S. Jannin, Nat. Commun., 2017, 8, 13975.

17 K. W. Lipsø, S. Bowen, O. Rybalko and J. H. ArdenkjærLarsen, J. Magn. Reson., 2017, 274, 65-72.

18 J. Jeener and P. Broekaert, Phys. Rev., 1967, 157, 232-240.

19 H.-M. Vieth and C. S. Yannoni, Chem. Phys. Lett., 1993, 205, 153-156.

20 N. D. Kurur and G. Bodenhausen, J. Magn. Reson., Ser. A, 1995, 114, 163-173.

21 S. Emid, J. Konijnendijk, J. Smidt and A. Pines, Physica B + C, 1980, 100, 215-218.

22 S. Zhang, E. Stejskal, R. Fornes and X. Wu, J. Magn. Reson., Ser. A, 1993, 104, 177-179.

23 A. K. Khitrin, J. Xu and A. Ramamoorthy, J. Magn. Reson., 2011, 212, 95-101.

24 J. Jeener, R. Du Bois and P. Broekaert, Phys. Rev., 1965, 139, A1959-A1961.

25 A. G. Redfield, Science, 1969, 164, 1015-1023.

26 D. E. Demco, J. Tegenfeldt and J. S. Waugh, Phys. Rev. B: Condens. Matter Mater. Phys., 1975, 11, 4133-4151.

27 M. Kunitomo, H. Hatanaka and T. Hashi, Phys. Lett. A, 1974, 49, 135-136.

28 J.-S. Lee and A. K. Khitrin, J. Chem. Phys., 2008, 128, 114504.

29 S. J. Elliott, S. F. Cousin, Q. Chappuis, O. Cala, M. Ceillier, A. Bornet and S. Jannin, Magn. Reson., 2020, 1, 89-96. 
30 A. Bornet, J. Milani, B. Vuichoud, A. J. Perez Linde, G. Bodenhausen and S. Jannin, Chem. Phys. Lett., 2014, 602, 63-67. 31 A. Bornet, A. Pinon, A. Jhajharia, M. Baudin, X. Ji, L. Emsley, G. Bodenhausen, J. H. Ardenkjær-Larsen and S. Jannin, Phys. Chem. Chem. Phys., 2016, 18, 30530-30535.

32 K. J. Harris, A. Lupulescu, B. E. J. Lucier, L. Frydman and R. W. Schurko, J. Magn. Reson., 2012, 224, 38-47.

33 P. Berthault, H. Desvaux, G. Le Goff and M. Pétro, Chem. Phys. Lett., 1999, 314, 52-56.
34 S. J. Elliott et al., Submitted, 2021.

35 P. Niedbalski, C. Parish, A. Kiswandhi, Z. Kovacs and L. Lumata, J. Phys. Chem. A, 2017, 121, 3227-3233.

36 L. Latanowicz, Concepts Magn. Reson., Part A, 2005, 27A, 38-53.

37 S. F. J. Cox, S. F. J. Read and W. T. Wenckebach, J. Phys. C, 1977, 10, 2917.

38 Q. Stern et al. In Press, 2021.

39 S. J. Elliott et al., In Preparation, 2021. 